\title{
MOVEMENT PARALLAX: AN ASYMPTOTIC FUNCTION OF AMPLITUDE AND VELOCITY OF HEAD MOTION
}

\author{
WOLFGANG HELL \\ Fachgruppe Psychologie, Universität Konstanz, 775 Konstanz/Germany
}

(Received 10 Decemher 1976; in revised form 21 July 1977)

\begin{abstract}
Three experiments providing a two-object display with monocular movement parallax acting as the only effective cue to depth were carried out with ten observers. The matched width of the variable (rear) object, i.e. the amount of constancy, was taken as a measure of the effectiveness of movement parallax under the condition under question. Experiment I showed that successively disparate images (position parallax) are not sufficient to produce the impression of depth as compared to movement parallax. In Experiment 2 and 3 velocity $(b)$ and amount $(d)$ of head movement were varied. At values 5-10 times threshold, depth perception by movement parallax reached half of its maximal effectiveness. It was virtually independent of $r$ and $d$ when these values exceeded 20 times threshold level.
\end{abstract}

Key words-movement parallax; monocular depth perception

\begin{abstract}
Although monocular movement parallax has been investigated intermittently since Bourdon's experiment (1898), there still exist studies questioning its role as a cue to depth. Observers perceived motion rather than depth in one study (Gibson et al., 1959) or simply separation in depth, relative distance being indeterminate in another (Smith and Smith, 1963). Gogel and Tietz (1974) have also reported apparent object motion concomitant with head motion, when the object's depth is not perceived corrcctly. And Eriksson (1972) found that in some cases even a reversal of apparent order in depth of two luminous objects occurred when viewed in total darkness.
\end{abstract}

Nevertheless movement parallax is widely acknowledged as a cue to relative or even absolute (Ferris. 1972; Johansson, 1973) depth. But though most authors agree that it works, few suggestions have been offered to explain how it does. The results of electrophysiology suggest a possible approach to the explanation of the effectiveness of movement parallax in depth perception. There exists strong evidence for the existence of cells in the visual system that respond to the direction and velocity of a moving stimulus (Barlow and Hill 1963a, 1963b, 1964; Bridgeman, 1972) and a hypothesis to explain space perception on that basis has been put forward (Nakayama and Loomis, 1974). The involvement of the motion detecting cells in the depth analysing process, in the case of retinal motions occurring with head motions (relative movement parallax), would be confirmed if it could be shown that successively disparate images (position parallax) are not sufficient for depth perception, but that motion flow over the retina is required. This will be investigated in Experiment 1 .

The most concrete and often cited definition of movement parallax has been presented by Graham (1965, p. 504): "When a subject's eyes move with respect to the environment, or when the environment moves with respect to a subject's eyes, a differential angular velocity exists between a line of sight to a fixated object and the line of sight to any other object in the visual field." It can be shown (Appendix 1) that for small head movements and the line of sight being roughly perpendicular to the direction of head movement

$$
\omega=v\left(\frac{1}{a}-\frac{1}{b}\right)
$$

and

$$
\Delta \gamma=d\left(\frac{1}{a}-\frac{1}{b}\right)
$$

where $a, b$ are the object distances, $v$ and $d$ velocity and amplitude of head movement, $\omega$ the differential angular velocity, and $\Delta \gamma$ the variation of the angle $\gamma$ between the two lines of sight (see Fig. 5).

If we consider $\omega$ the critical value for movement parallax, as Graham's definition seems to imply, the effectiveness of movement parallax is enhanced by a greater velocity of relative movement of the observer and the objects. This is apparently true for threshold conditions (Graham et al., 1948; Zegers, 1948), but in supra-threshold conditions normal life experience teHs us that depth perception by monocular movement parallax should not depend on either the amplitude or the velocity of head movement over a considerably wide range, and the expression $\omega / v=$ $1 / a-1 / b$ that depends only on object distances seems to be a better representation of what is going on in depth perception by movement parallax.

This can be tested by experiments in which monocular movement parallax is the only cue to depth and the assumptions underlying the derivation of (1) are valid. There are only limited data on the influence of velocity $(v)$ and amplitude $(d)$ of head movement. Although Zegers reports that the threshold angular velocity for a motionless observer to perceive the depth difference of two needles moving along a horizontal path "increase(s) with an increase in basic rate of stimulus movement up to a limiting rate" (Zegers, 1948 , p. 497), no attempt has been made to vary $v$ systematically under conditions of active head movement of the observer. 


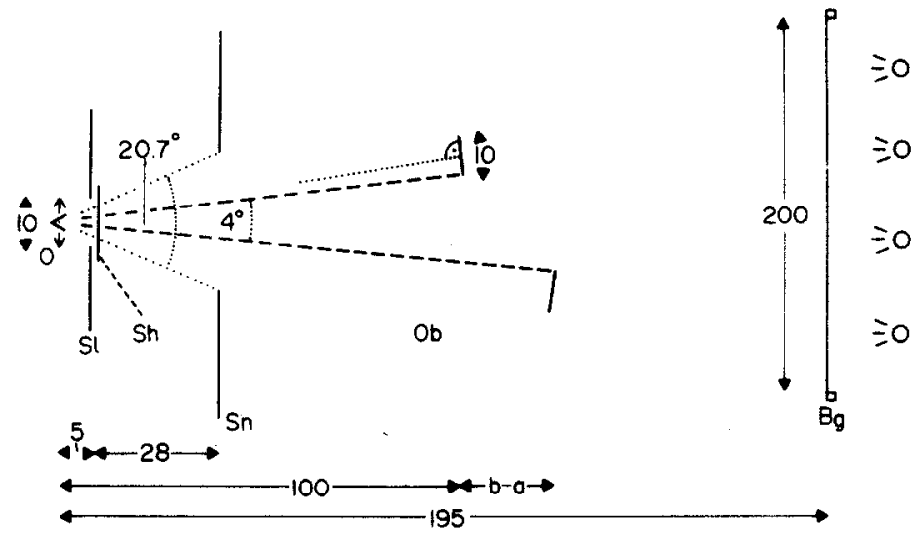

Fig. 1. Schematic top-view drawing of the experiment, showing observer (O), slit (Sl), shutter (Sh), screen $(\mathrm{Sn})$, objects $(\mathrm{Ob})$, and background $(\mathrm{Bg})$. All distances are given in $\mathrm{cm}$.

Verwey (1909) determined the threshold for $d$ necessary to perceive a relative movement of two objects. In the case of active head movement Redding et al. (1967) came to the conclusion that "small" head sways $(20 \mathrm{~cm})$ do not improve depth perception compared to a no-movement condition while "large" body sways $(46 \mathrm{~cm})$ do. On the other hand Cords $(1913$. $1913 / 14)$ found that "with head movements from 9.5 to $3 \mathrm{~cm}$ depth perception (by movement parallax) is nearly constant..., it deteriorates rapidly from 3 to $1 \mathrm{~cm}$, and nearly ceases with head movements below $1 \mathrm{~cm}$ " (Cords $1913 / 14$, p. 421). Since published data on the influence of amplitude and velocity of head movement are either contradictory, insufficient or non-existent, Experiments 2 and 3 study the influence of $v$ and $d$ on movement parallax as a cue to depth.

In all three experiments the matched width of the variable (rear) of the two objects has been taken as a measure of the effectiveness of movement parallax. When no cues to depth are available the perceived size of objects is determined by the retinal image sizes (law of the visual angle). In a full cue situation, on the other hand, objects of equal physical size are perceived over a wide range as being equally large irrespective of their different distances or retinal image sizes (size constancy law; Graham, 1965, pp. 506-507). Thus matched size obtained in a limited cue situation, as in the experiments to be reported, can be regarded as an indicator of the effectiveness of the available cues. In this case, in which visible cues were limited to movement parallax, and the more distant of two objects was matched to the nearer one, the smaller (larger) the matched variable object, the greater (smaller) is the effectiveness of movement parallax.

\section{METHOD}

\section{Ohservers}

Six female and four male students served as paid subjects in all three experiments. The normal or corrected visual acuity in their dominant eye was at least $20 / 20$.

\section{Apparatus}

The apparatus was designed to provide the observer with a visual field of two black rectangular objects in front of a bright patterned background (Fig. 1). The observer sat in a booth lined with black cloth on an adjustable chair in front of a $10-\mathrm{cm}$ wide horizontal slit (SI). For fixation of the head he bit into a bite-board mounted on a trolley which could be rolled laterally with the observer's head movement along a 16-cm horizontal track. The height of the bite-board could be adjusted to center the observer's dominant eye vertically on the slit. at a distance of $5 \mathrm{~cm}$. The range of lateral motion $(d)$ could be varied from 0 to $10 \mathrm{~cm}$ by stops positioned on the track. A force of 0.3 newton was necessary to keep the trolley moving. The slit was closed by an electromechanical shutter (Sh). The motion of the trolley operated a tooth-gear that was connected to a tacho-alternator. Two thresholds could be chosen between 0 and $100 \mathrm{~cm} / \mathrm{s}$ so that the shutter only opened in a preselected range depending on the respective $v$. The opening times were summated to yield a total opening time of $20 \mathrm{~s}$ per trial. The visual field of the motionless observer was restricted to $4.3^{\circ}$ high by $20.7^{\circ}$ wide by a screen (Sn) positioned $33 \mathrm{~cm}$ from the observer's eye.

The objects $(\mathrm{Ob})$ consisted of thin black plastic plates. beveled at the edges, $30 \mathrm{~cm}$ in height, and mounted on movable supports so that neither their upper nor lower end was visible. The width of the standard (nearer) object was $10 \mathrm{~cm}$. The variable object consisted of three plates, two of them sliding over the middle one so that the total width could be varied from 8 to $12.3 \mathrm{~cm}$ either by the experimenter or by the observer turning a knob connected to a flexible cable leading to the object. The objects were oriented perpendicularly to the observer's line of sight when his eye was centered on the slit. The angular separation of the inner edges of the objects was 4 , when the width of the variable object was $10 \mathrm{~cm}$. The standard object was positioned at a constant distance of $a=100 \mathrm{~cm}$. the variable object at either $b=110 \mathrm{~cm}$ or $b=120 \mathrm{~cm}$.

The background $(\mathrm{Bg})$, covering the entire visual field. consisted of stretched translucent paper $(200 \times 50 \mathrm{~cm})$ at a distance of $195 \mathrm{~cm}$ from the observer's eye. It was rearilluminated by four fluorescent lamps whose brightnesses were adjusted to yield a constant illuminance of 14 lumen $/ \mathrm{m}^{2}$. The background was regularly patterned with black spots of $0.4 \mathrm{~cm}$ diameter separated by $3 \mathrm{~cm}$ center-tocenter, in order to present a more natural situation. The ceiling. the table on which the object stands rested, and the rear side of the screen were covered with black velvet to eliminate brightness differences of the objects due to stray light. Two control experiments with two additional observers made sure that neither auditory nor visual cues besides movement parallax and relative sizes of the objects were effective.

\section{Procedure}

The naive observer was first informed that "movement 
parallax are the apparent motions that appear between two objects at different distances. if you move your head" and that it "might contribute to the perception of depth differences". Then his non-dominant eye was occluded with an eye patch, eye height and amplitude of head movement were adjusted, and he was dark adapted for at least five minutes. The observer was told that he would see two objects and that his task was "to vary the width of the variable object so that you perceive both objects as being subjectively equally wide". The observation time (total shutter opening) during each trial was $20 \mathrm{~s}$. Before each trial the observer was told which would be the variable object ("left", "right"). Whenever the experiment was begun or resumed after dark adaptation, the observer's first eight settings were not recorded in order to exclude adaptation effects. Whenever the experimental conditions were changed, the first four settings were omitted, in both cases without the observer's knowledge. The interval between measurements was about $1 \mathrm{~min}$.

In all three experiments the positions (left, right) of the objects, the initial width of the variable object $(9.1,9.7$, $10.3,10.9 \mathrm{~cm})$, and the relative distance $(b-a=10,20 \mathrm{~cm})$ were varied randomly with the restriction that every combination of position and initial width occurred twice for every combination of $b-a$ and experimental condition. Therefore 16 measurements per data point were made with each observer. The succession of the different conditions was varied among the observers. All observers first participated in Experiment 1, then in the remaining two experiments in random order.

The recorded variable was the matched width of the variable (rear) object. In addition, both the average and the maximal velocities of head movement were recorded. Whenever the observer was not ready or not satisfied with his final adjustment or in cases of apparatus failure, the measurement was repeated without the observer's knowledge after the next trial.

\section{EXPERIMENTS}

\section{Experiment 1}

Experiment 1 consisted of three conditions: head fixed, position parallax and movement parallax. During the condition "head fixed" the shutter was opened only when the observer was held motionless on the bite-board by locking the trolley between the two stops on the track. Under the condition "position parallax" the tachoalternator was adjusted so that the shutter closed whenever $v$ exceeded $1 \mathrm{~cm} / \mathrm{s}$. The observer was told to use the total range of $10 \mathrm{~cm}$ to make fast movements interrupted by "stand-stills" of about $1 \mathrm{~s}$ duration at whatever position he liked. He was requested to use the two extreme positions as well as others in between. In the "movement parallax" condition $d$ was $10 \mathrm{~cm}$ and there was no restriction of $v$. The observer was told to use the total range with as little interruption as necessary for change of direction and to choose whatever velocity he liked but to move at as nearly the same velocity as possible from trial to trial.

\section{Experiment 2}

This experiment consisted of four different conditions, i.e. four different maximal velocities of head movement $(v=0,3,6 \mathrm{~cm} / \mathrm{s}$ and $a d(l b)$. The $v=0 \mathrm{~cm} / \mathrm{s}$ condition was identical to the head fixed condition of Experiment 1. For the 3- and $6-\mathrm{cm} / \mathrm{s}$ conditions an upper velocity limit of 3 respectively $6 \mathrm{~cm} / \mathrm{s}$ was preselected, the shutter closing during velocities in excess of the preselected maximum and remaining closed until $v$ was reduced. The observer was instructed to use the total range $(d=10 \mathrm{~cm})$ and to move regularly as near to the velocity limit as possible. Prior to the beginning of the experiment he was permitted to try out different velocities as long as he wished, but without benefit of view of the stimulus objects. The shutter opened after the start signal with the observer's first movement. Because the $3-\mathrm{cm} / \mathrm{s}$ condition allowed for only a few (about 3 ) changes of direction, the observer was told under this condition to start every time at the same extreme position. The ad lib condition was identical to the movement parallax condition of Experiment 1.

\section{Experiment 3}

Five different amounts of head movement $(d=0$, $1.5,3,6,10 \mathrm{~cm})$ were the conditions in Experiment 3. The $0-\mathrm{cm}$ condition again was identical to the head fixed condition of Experiment 1. For the other conditions $d$ was restricted to the respective values the stops on the track being positioned so as to ensure that the eye was midline between the objects, when the trolley was at the middle position of the track. The observer was requested to use the total range of $d$, and was reminded occasionally to do so. $v$ was $a d ~ l i b$ and the observer was instructed to move at as nearly the same velocity as possible from trial to trial.

\section{RESULTS}

\section{General results}

For all three experiments a three-way analysis of variance was carried out. In all cases the influence of relative depth $(b-a=10,20 \mathrm{~cm})$ was highly significant, $F(1,9)=1179$ (Experiment 1), 1004 (Experiment 2), 890 (Experiment 3), $p<0.001$, as was the interaction of relative depth with motion state, $F(2,18)=14.7($ Experiment 1$)$, velocity, $F(3,27)=10.0$ (Experiment 2), and amplitude of head motion, $F(4,36)=18.1$ (Experiment 3$), p<0.001$ in all cases. It therefore follows that the data for $b-a=10 \mathrm{~cm}$ show a stronger approach to constancy $(10 \mathrm{~cm}$ width) in absolute values than the data for $b-a=20 \mathrm{~cm}$.

The influence of motion state, velocity, and amplitude of head motion was significant in all experiments, $F(2,18)=29.6($ Experiment 1$), F(3,27)=32.0$ (Experiment 2), $F(4,36)=39.3 \quad$ (Experiment 3), $p<0.001$ in all cases, as was the interaction with the observers, $F(18,18)=7.4 \quad$ (Experiment 1), $F(27,27)=4.7$ (Experiment 2$), F(36,36)=6.5$ (Experiment 3), $p<0.001$ in all cases. It therefore can be concluded that the experimental conditions had a marked influence on the results but also that great individual differences existed in the effectiveness of movement parallax.

The matched widths for head fixed and position parallax didn't reach the expected values $(11$ respectively $12 \mathrm{~cm}$ ) for equal retinal image size. This can be attributed to the initial retinal image sizes acting as a cue to depth, shown by the fact that the observer's final adjustments were clearly dependent on the initial settings of the width of the variable object by the experimenter. The smaller the initial width of the variable (rear) object, the smaller was the adjusted width. A similar dependency in the movement conditions could not be found. 


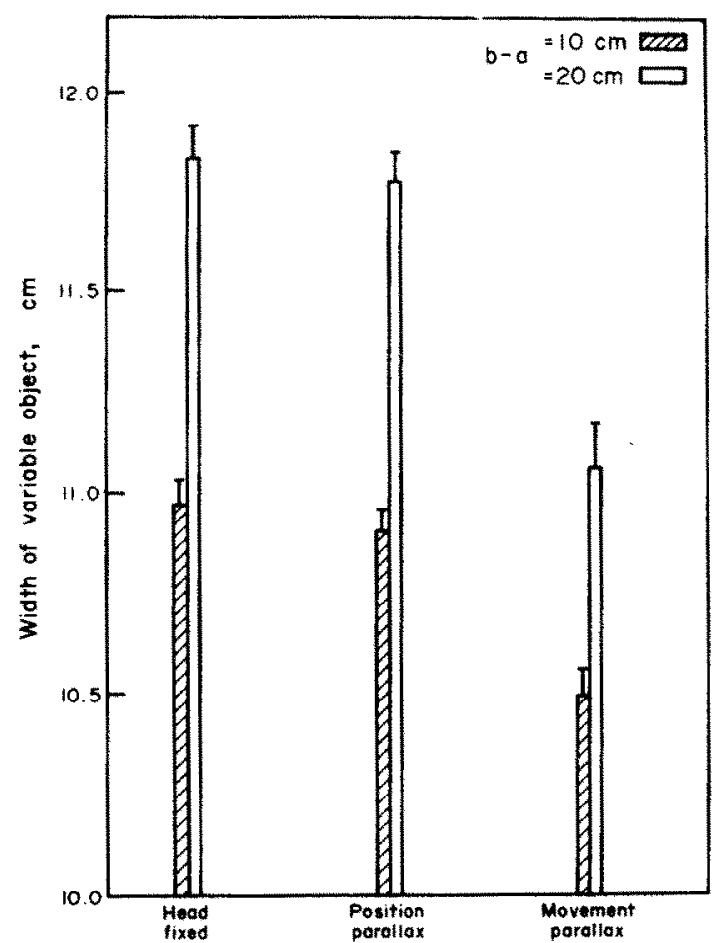

Fig. 2. Matched width of the variable (rear) object under the conditions head fixed, position parallax, and movement parallax for two relative object distances $(b-a=10$. $20 \mathrm{~cm}$ ). Standard object width $10 \mathrm{~cm}$, average (maximal) velocity of head motion in the movement parallax condition $8.2(15.3) \mathrm{cm} / \mathrm{s}$. average opening time under position parallax $1.04 \mathrm{~s}$. Standard errors are indicated.

\section{Experiment 1}

The averaged values over the ten observers are shown in Fig. 2. The application of the Tukey (b) test for the two relative distances separately resulted in a significant difference, $q<0.01$, between movement parallax and both position parallax and head fixed, and a nonsignificant difference, $q>0.05$. between position parallax and head fixed. This clearly demonstrates that successively disparate images (position parallax) are not sufficient to produce depth perception. The slight decrease of the values for position parallax as compared to the values for the head fixed condition can be explained by the fact that apparatus limitations didn't allow the closure of the shutter but for velocities exceeding $1 \mathrm{~cm} / \mathrm{s}$.

\section{Experiment?}

Figure 3 shows the averaged results for the investi. gation of the influence of velocity of head movement on the effectiveness of movement parallax. The data of both distance conditions have been fitted ( $F$-Test of goodness of fit: $F<1$ ) with hyperbolas of the type $y=x /(n x+m)$ shifted to the appropriate place on the coordinate system. Though the $F$-values are fairly small (as would be expected for a curve varying only over a $1-\mathrm{cm}$ range $10 \mathrm{~cm}$ above the abscissa) the fits cannot be regarded as fully satisfactory. Both functions overestimate the decrease between $v=6 \mathrm{~cm} / \mathrm{s}$ and the ad lib maximal velocity $(15.7 \mathrm{~cm} / \mathrm{s})$ and underestimate the decrease between $v=3 \mathrm{~cm} / \mathrm{s}$ and $v=$ $6 \mathrm{~cm} / \mathrm{s}$. Using the same data it could also be argued that a relatively sharp decrease takes place around $v=3 \mathrm{~cm} / \mathrm{s}$ leading to a plateau commencing at about $v=6 \mathrm{~cm} / \mathrm{s}$. It should be noted that the greatly vary-

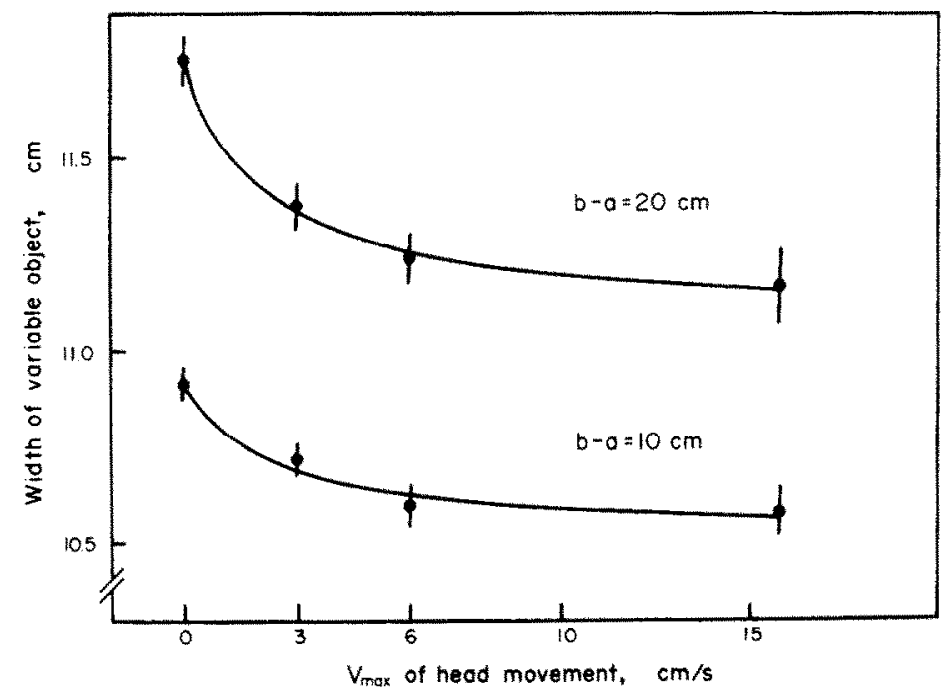

Fig. 3. Dependence of the matched width of the variable (rear) object upon the maximal velocity of the observer's head motion. Standard object width $10 \mathrm{~cm}$. average velocities of head motion $1.9 \mathrm{~cm} / \mathrm{s}$ $\left(v_{\max }=3 \mathrm{~cm} / \mathrm{s}\right), 3.7 \mathrm{~cm} / \mathrm{s}\left(v_{\max }=6 \mathrm{~cm} / \mathrm{s}\right)$. and $8.9 \mathrm{~cm} / \mathrm{s}\left(v_{\operatorname{mix}}=15.7 \mathrm{~cm} / \mathrm{s}\right)$. The curve for $b-a=10 \mathrm{~cm}$ is fitted with

$$
\left.y=\frac{x+2.5}{0.3962(x+2.5)-0.1346}+8 \text { (asymptote: } 10.52\right)
$$

and the curve for $b-a=20 \mathrm{~cm}$ is fitted with

$$
y=\frac{x+3}{0.4829(x+3)-0.3576}+9 \text { (asymptote: } 11.07 \text { ). }
$$

Standard errors are indicated. 
ing individual ad lib maximal velocities $(9.2-20.6 \mathrm{~cm} / \mathrm{s})$ correlated neither with the individual decrease of object size match from $v=6 \mathrm{~cm} / \mathrm{s}$ to the respective ad $l i b$ velocity nor with the individual effectiveness of movement parallax as might have been expected.

A possible methodological artifact consists of the frequent shutter closures occurring in the 3- and $6-\mathrm{cm} / \mathrm{s}$ conditions, which might have disrupted the perceptual act and in any case prolonged the over-all trial time, since only the opening times contributed to the total trial duration of $20 \mathrm{~s}$. Without this disturbance the values of the results of these two conditions might well have been smaller (i.e. greater constancy). Another uncontrolled variable is the surely different velocity-time functions under the different $v$ 's that show in the different ratios of maximal to average velocity (compare legend to Fig. 3). Nevertheless two conclusions can be drawn: (1) The effectiveness of movement parallax reaches a maximum which, under the experimental conditions used, lies about halfway between no effect and full constancy. (2) More than half of the maximal effect (asymptotic value) of movement parallax appcars at $r=3 \mathrm{~cm} / \mathrm{s}$ while there is scarcely any further improvement beyond $v=6 \mathrm{~cm} / \mathrm{s}$.

\section{Experiment 3}

The results of the variation of the amplitude of head movement are plotted in Fig. 4. The fit with the hyperbolas is quite satisfactory $(F$-test of goodness of fit: $F<1$ ). The average and maximal velocities differed over the conditions as well as the ratios between them. But this variation is not critical since the lowest maximal velocity $(v=7.8 \mathrm{~cm} / \mathrm{s}$ for $d=$
$1.5 \mathrm{~cm}$ ) exceeds the $6 \mathrm{~cm} / \mathrm{s}$ condition of Exp. 2, which proved to differ but slightly from the ad lib condition $(v=15.7 \mathrm{~cm} / \mathrm{s})$. Thus the differing velocities fall into a range where the influence of $v$ can be neglected.

It can be concluded that the effectiveness of movement parallax quickly approaches a maximum lying about halfway between no effect and full constancy (see the asymptotic values given in the legend to Fig. 4), under the experimental conditions used. The midpoint of the hyperbolas between the start $(d=$ $0 \mathrm{~cm}$ ) and the asymptote is reached at $d=1.09 \mathrm{~cm}$ $(b-a=10 \mathrm{~cm})$ and $d=1.26 \mathrm{~cm}(b-a=20 \mathrm{~cm})$. It can be argued that movement parallax at these values reaches half of its final effectiveness.

\section{DISCUSSION}

If one assumes that the matched width of the variable object represents the amount of constancy $(n)$ that can be reached under a given condition, one can calculate, in a slight modification of Brunswik's ratio, the percentage of $n$ as

$$
n=100 \frac{A b-a B}{A(b-a)}
$$

where $a, b$ are the distances of the two objects and $A, B$ their widths. For the asymptotic values averaged over Experiment 2 and 3 one obtains $n=48.5 \%$ $(b-a=20 \mathrm{~cm})$ and $n=51 \% \quad(b-a=10 \mathrm{~cm})$. Though the absolute values of the matched widths under the two relative distances differ, the amount of constancy is the same. This also holds for other

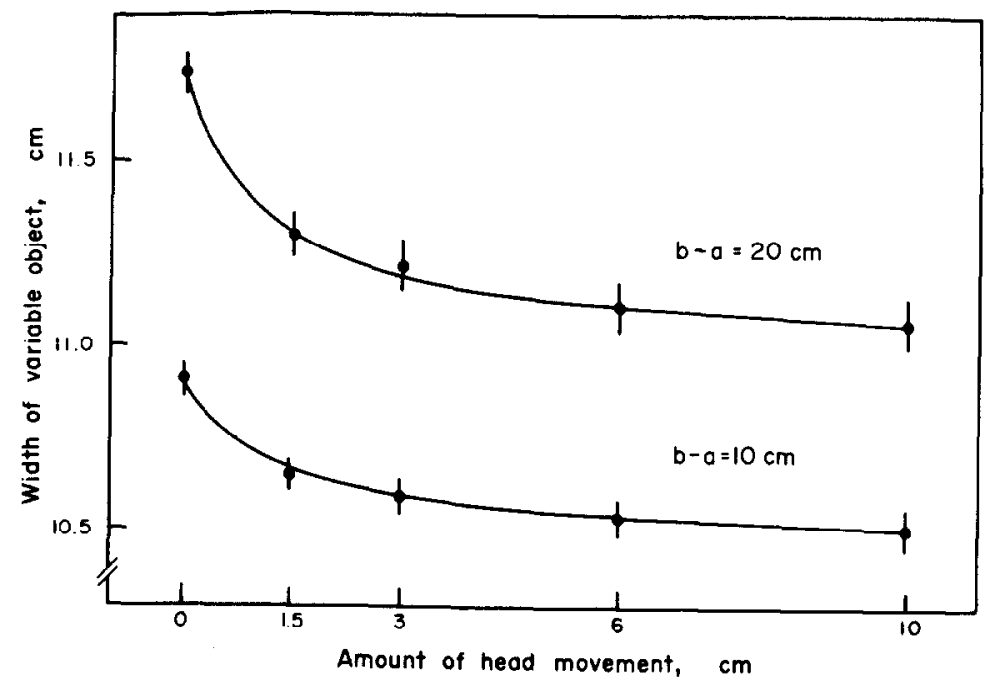

Fig. 4. Dependence of the matched width of the variable (rear) object upon the amplitude of head motion. Standard object width $10 \mathrm{~cm}$, average (maximal) velocities (in $\mathrm{cm} / \mathrm{s}$ ) from $d=1.5 \mathrm{~cm}$ to $d=10 \mathrm{~cm}: 2.7(7.8), 4.5(10.3), 7.5(13.8), 10.5(17.4)$. The curve for $b-a=10 \mathrm{~cm}$ is fitted with

$$
\left.y=\frac{x+1.5}{0.4060(x+1.5)-0.0917}+8 \quad \text { (asymptote: } 10.52\right)
$$

and the curve for $b-a=20 \mathrm{~cm}$ is fitted with

$$
y=\frac{x+1.5}{0.5034(x+1.5)-0.2093}+9 \quad \text { (asymptote: } 10.99 \text { ), }
$$

Standard errors are indicated. 
conditions. The maximal amount of constancy with movement parallax as the only cue to depth is about $50 \%$ in the experimental conditions used.

Experiments 2 and 3 were designed to determine whether movement parallax as a cue to depth depends on the velocity $(w)$ and the amplitude of head movement $(d)$. For both cases we can assume that it does, at least for small values of $r$ and $d$, but it seems to be more and more independent for increasing values of $t$ and $d$. Whereas $r$ increases from 6 to $15.7 \mathrm{~cm} / \mathrm{s}$ and the differential angular velocity more than doubles, the amount of constancy reached only improves from $38,40^{\circ},(b-a=20,10 \mathrm{~cm})$ to $42^{\circ}$.

It is interesting to compare $\omega$ with the $\omega$-values given by Graham et al. (1948). Since the conditions are different the comparison can only be a rough one. Let us therefore take $\omega_{t}=100 \mathrm{arcs} / \mathrm{s}$ as a representative threshold value from their data. In the present experiment $\omega$ is found to be $560 \mathrm{arcs} / \mathrm{s}(b-a=$ $10 \mathrm{~cm})$ respectively $760 \mathrm{arcs} / \mathrm{s}(b-a=20 \mathrm{~cm})$, calculated from (1) with the respective $v$ midway between $v=0 \mathrm{~cm} / \mathrm{s}$ and the asymptotical $v$. i.e. $v=3 \mathrm{~cm} / \mathrm{s}$ respectively $v=2.2 \mathrm{~cm} / \mathrm{s}$. It can be concluded that at an $\omega$-value as small as about 5-10 times larger than threshold movement parallax reaches half of its maximal effect. From $\omega$-values of about 20 times larger than $\omega_{t}(\omega)=19 \mathrm{arcmin} / \mathrm{s}$ for $v=6 \mathrm{~cm} / \mathrm{s}$ and $b-a=$ $10 \mathrm{~cm}$ ) on movement parallax is widely independent of $v$. Therefore at values greatly exceeding threshold, $\omega / v=1 / a-1 / b$ seems to be a better representation of what is happening since its value depends only on the geometry of the situation.

Similar conclusions can be drawn for the variation of $d$. With the midpoints of the fitted hyperbolas at $d=1.09(b-a=10 \mathrm{~cm})$ and $d=1.26 \mathrm{~cm}(b-a=$ $20 \mathrm{~cm}$ ) we get from (2) $\Delta y=240$ arcs and $\Delta y=360$ ares. The only data available for comparison are Verwey's (1909), who found for example for two objects at 29 and $30 \mathrm{~cm}$ absolute distance, that $d=2 \mathrm{~cm}$ is the threshold for the perception of movement parallax. corresponding to a variation of the visual angle between the objects $\Delta y=47$ arcs. Since he used only one subject (himself), the comparison can be considered merely suggestive that, when $d$ is 5-10 times larger than threshold, the resulting size constancy reaches half of its final effect. At greater values of $d$ depth perception by movement parallax becomes largely independent of the amount of head movement. To determine the exact dependency of movement parallax on $v$ and $d$ near threshold would require a measurement of the thresholds under identical conditions.

Experiment 1 suggests that motion detectors may process the information provided by relative movement parallax. But this information is ambiguous. since the differential angular velocity between two objects that elicits the cell response depends on both $v$ and $a, b$. Two theories exist to explain depth perception on the basis of differing retinal image motions. Nakayama \& Loomis (1974) suggest a physiological mechanism that extracts "distinct optical surfaces" from the motion pattern of the optical input. But this system only explains relative depth perception. Eriksson $(1974$, p. 225) postulates an "interaction between the optical system and information from the bodystate system" yielding correct depth perception, except in the case of passive zero acceleration motions.

In the normal visual situation (sitting in a train. for instance) redundant depth information in addition to movement parallax is available. As soon as one absolute distance is known, movement parallax information ceases to be ambiguous and the motion-state is known. It can be thought of the visual system extracting from the optical input a reference at "infinity" (e.g. the slowest appearing angular velocity). accounting for the relative velocity compared to this point in order to process values proportional to $\omega / t$, and comparing these values to the $\omega / t$-value for the reference in order to get veridical information about apparent object distances.

Such a view demands that in a situation of passive movement parallax (stable observer, moving visual field), with no other cues to depth available, a rather poor depth perception exists. This is supported by the results of Gibson et al. (1959) and Smith and Snith (1963). The failure of these experiments to yield depth perception by motion parallax consists in the insufficient or ambiguous motion-state information of the observer.

Acknowledgement:-The research reported in this article was supported by Graduate Fellowship No. 15/00183 of the Federal Republic of Germany to the author and by Research Grant No. 33/73 of the University of Konstanz to R. B. Freeman, Jr., whose continuous support and helpful comments on this paper are gratefully acknowledged.

\section{REFERENCES}

Barlow H. B. and Hill R. M. (1963a) Selective sensitivity to direction of movernent in ganglion cells of the rabbit retina. Science $139,412-414$.

Barlow H. B. and Hill R. M. (1963b) Evidence for a physiological explanation of the Waterfall Phenomenon and figural after effects. Nature 200, 1345-1347.

Barlow H. B. and Hill R. M. (1964) Retinal ganglion colls responding selectively to direction and speed of image motion in the rabbit. J. Physiol. 173, 377-407.

Bridgeman B. (1972) Visual receptive fields sensitive to absolute and relative motion during tracking. Science 178, $1106-1108$

Bourdon B. (1898) La perception monoculaire de la profondeur. Rerue Philosophique 46, 124-145.

Cords R. (1913) Der Einfluß der parallakt ischen Verschiebung auf die Tiefenwahrnehmung. Klin. Mbl. Augenheilk. 51. 421 .

Cords R. (1913/14) Bemerkungen zur Untersuchung des Tiefenschätzungsvermögens. III. Die Verwertung der parallaktischen Verschiebung durch Einäugige. $Z$. Augenheilk. 30, 34-46.

Eriksson E. S. (1972) Movement parallax, Anisotropy, and Relative Size as Determinants of Space Perception Report 13I, University of Uppsala, Uppsala. Sweden.

Eriksson E. S. (1974) A theory of veridical space perception. Scand. J. Psychol. 15, 225-235.

Ferris S. H. (1972) Motion parallax and absolute distance. J. exp. Psychol 95, 258-263.

Gibson E. J., Gibson J. J., Smith O. W. and Flock H. (1959) Motion parallax as a determinant of perceived depth. J. exp. Psychol. 58, 40-51.

Gogel W. C. and Tietz J. D. (1974) The effect of perceived distance on perceived movement. Perc. \& Psychoph. 13, $284-292$. 
Graham C. H. (1965) Vision and visual perception. J. Wiley \& Sons.

Graham C. H., Baker K. E., Hecht M. and Lloyd V. V. (1948) Factors influencing thresholds for monocular movement parallax. J. exp. Psychol. 38, 205-223.

Johansson G. (1973) Monocular movement parallax and near space perception. Perception 2, 135-146.

Nakayama K. and Loomis J. M. (1974) Optical velocity patterns, velocity-sensitive neurons, and space perception: a hypothesis. Perception 3, 6380.

Redding G. M., Mefferd R. B. and Wieland B. A. (1967) Effect of observer movement on monocular depth perception. Percept. \& Mot. Skills. 24, 725-726.

Smith O. W. and Smith P. C. (1963) On motion parallax and perceived depth. J. exp. Psychol. 65, 107-108.

Verwey A. (1909) Het Zien van Diepte door Middel der éénoogige Verschuiving. Nederl. Tijdschrift $v$. Geneeskunde 2, 1808-1816.

Zegers R. T. (1948) Monocular movement thresholds as functions of field size, field position, and speed of stimulus movement. J. Psychol, 26, 477-498.

\section{APPENDIX}

We calculate the differential angular velocity $(\omega)$ in the case where the two objects and the observer's line of movement lic in the same horizontal plane (Fig. 5). When $\gamma=\beta-\alpha, \gamma^{\prime}=\beta^{\prime}-x^{\prime}, \tan \alpha=a / a^{\prime}, \tan x^{\prime}=a /\left(a^{\prime}+d\right), \tan$ $\beta=b /\left(b^{\prime}-d\right), \tan \beta^{\prime}=b / b^{\prime}$, we obtain:

$$
\begin{aligned}
\Delta \gamma=\arctan \frac{b}{b^{\prime}}+\arctan \frac{a}{a^{\prime}} & -\arctan \frac{b}{b^{\prime}-d} \\
& -\arctan \frac{a}{a^{\prime}+d}
\end{aligned}
$$

Although the general case is more complicated, we will assume that $a^{\prime}, b^{\prime}, d \ll a, b$, i.e. the line of sight is roughly perpendicular to the direction of head motion, and the amplitude of head motion is small compared to the object distances. These restrictions are generally fulfilled for a non-locomoting observer moving his head or body laterally and usually not for a locomoting observer. Under the

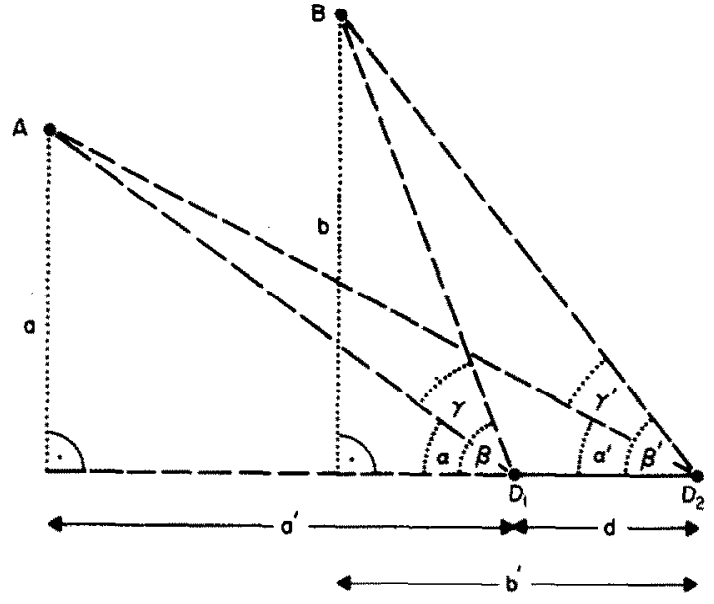

Fig. 5. Geometry of movement parallax in the case of two objects $(A, B)$ and an observer moving from $D_{1}$ to $D_{2}$ $\left(A, B, D_{1}, D_{2}\right.$ lie in the same plane)

above assumptions we can take advantage of the power series expansion of $\arctan x$,

$$
\arctan x= \pm \frac{\pi}{2}-\frac{1}{x}+\frac{1}{3 x^{3}}-\cdots|x|<1
$$

where we can, with small error, drop all but the first two terms, and get

$$
\Delta y=d\left(\frac{1}{a}-\frac{1}{b}\right)
$$

$\omega / \Delta y$ is yielded by differentiation of $\omega / \Delta y$ with respect to time.

$$
\omega=v\left(\frac{1}{a}-\frac{1}{b}\right)
$$

All the above considerations are equally valid when $v$ is assumed to be the relative velocity of laterally moving objects with respect to a stationary observer.

Zusammenfassung-Drei Experimente mit monokularer Bewegungsparallaxe als einzig wirksamem Tiefenwahrnehmungsparameter in einer Versuchsanordnung mit zwei Objekten wurden mit zehn Versuchspersonen durchgefïhrt. Die eingestellte Breite des variablen (hinteren) Objektes, d.h. der Grad der Konstanz, diente als $\mathrm{Ma} B$ für die Wirksamkeit der Bewegungsparallaxe unter der jeweiligen Bedingung. Exp. 1 zeigte, daB die Aufeinanderfolge disparater Bilder (Stellungsparallaxe) im Vergleich mit Bewegungsparallaxe nicht genügt, um Tiefeneindruck zu erzeugen. In Exp. 2 und 3 wurden Geschwindigkeit (v) und AusmaB (d) der Kopfbewegung variiert. Tiefenwahrnehmung durch Bewegungsparallaxe erreichte die Hälfte der maximalen Wirksamkeit bei 5-10 fach überschwelligen Werten und war praktisch unabhängig von $v$ und $d$ ab 20 fach überschwelligen Werten 